\title{
Damage Detection in a Plate Structure based on FBG sensing Technique under a Single-Mode Lamb Wave
}

\author{
https://doi.org/10.3991/ijoe.v14i07.8965 \\ Cai Li $(\square)$ \\ Wuhan Donghu University, Wuhan, China \\ caili_whute163.com \\ Huang Xiao \\ Wuhan University of Technology, Wuhan, China \\ Zuo Xiaoqiong \\ Wuhan Donghu University, Wuhan, China
}

\begin{abstract}
Lamb wave is widely acknowledged as one of the most encouraging tools for damage identification in plate structures, and relevant research has been conducted intensively. However Lamb wave modes have different wave structure, frequency dispersion and attenuation characteristics, which are sensitivity to different types of damages and it is difficult to solve such engineering problems by conventional techniques. Although the single pattern detection method has been researched by piezoelectric wafers, there is little research about FBG sensing detection under single-mode ultrasonic Lamb wave technique currently. So this paper puts forward a single-mode Lamb wave technique for crack detection based on Fiber Bragg Grating sensor, which is used to receive the waves in the plate. First of all, measuring principle of single-mode ultrasonic Lamb technique and demodulation principle of the FBG sensor are introduced. And simulation analysis in the acoustic field is devoted, whose results lay the foundation for the damage detection in the plate. Then, the experimental system is built by a single-mode Lamb wave excitation, and the feasibility of fiber Bragg grating sensors in single-mode excitation method is verified by experiments.
\end{abstract}

Keywords_Lamb Wave; Damage Detection; Fiber Bragg Grating(FBG)

\section{Introduction}

Ultrasonic Lamb wave technology is one of the active structural health monitoring method, which is especially suitable for damage detection and health monitoring of plate structure because of its advantages, such as low-cost, long-range inspection, and testing inaccessible or fast scanning capabilities [1]. But ultrasonic guided wave modes have different wave structure, frequency dispersion and attenuation characteristics, which are sensitivity to different types of damages [2] [3]. So we should choose the appropriate mode for damage detection. Recently, some scholars have studied the 
methods of the single mode Lamb wave. Su et al. arranged two sensors in the upper and lower surfaces of the plate like structure, and obtained a relatively single mode Lamb wave by enhancing a certain mode and weakening the other modes. They have used this method to detect the delamination in the isotropic laminates[4]. Clarke et al. studied a new type of piezoelectric sensor by adding the front liner matching layer and the backing layer respectively in the front and back of a circular piezoelectric chip to make the piezoelectric sensor capable of motivating a single modality at low frequencies [5]. Now there are many studies on signal extraction, such as Wang etc. obtained damage index of ultrasonic guided wave in each propagation path based on correlation analysis and the defect imaging map represented by probability values, and realized positioning and identification of artificial defects in the plate [6]. Most damage detection and localization methods that have been proposed are based upon comparing monitored signals to baselines recorded from the structure prior to initiation of damage. Michaels proposed a two-step process whereby damage is first detected and is then localized and characterized, taking into account any conditions other than damage that have changed the ultrasonic signals [7]. Xiaole Liu presented a new model with periodic potential to induce the adaptive model, by which the system parameters were simultaneously optimized. In order to eliminate interference to obtain more useful information, the signals were preprocessed by Hilbert transform and High-pass filter before being input to the adaptive system and the improved signal-tonoise ratio was set as the evaluation index [8]. Aldo Baccigalup investigated Huang Hilbert Transform for evaluating the instantaneous frequency evolution in non-linear systems by comparing the different traditional approaches. In order to evaluate its performance, the proposed approach had been firstly applied to a synthesized signal with known instantaneous amplitude and frequency evolution [9]. Irene Buj-Corral studied acoustic emission by comparing two methods to treat the sound signal emitted during the machining process: Fast Fourier Transform and Hilbert Huang Transform [10]. But most of these methods are based on the piezoelectric film to motivate and receive. The attempt of the fiber grating in the single mode scheme is still rare.

Therefore, this paper aims to present an FBG sensing system based on a singlemode ultrasonic Lamb wave technique for the board plates. And the key contribution is that the single-mode ultrasonic Lamb wave technique-FBG sensing system is given. It can establish a detection method by combining the advantages of fiber grating sensing with ultrasonic Lamb wave technique. This paper is structured as follows. Section 2 reviews measuring principle of single-mode ultrasonic Lamb technique and demodulation principle of the FBG is given. Section 3 is devoted to simulation analysis in the acoustic field of the plate at different time or on different points, whose results lay the foundation for the damage detection in section 4 . On this basis, the experimental system is built and the responses under the single-mode excitation are carried out, and the feasibility of fiber Bragg grating sensors in single-mode excitation method is verified by experiments. Section 5 summarizes the full text. 


\section{Excitation and reception principle}

\subsection{Excitation principle of Lamb wave}

Lamb wave on the plate structure has many modal characteristics, containing at least two kinds, namely basic symmetric mode and basic antisymmetric mode. It can be seen from the figure 1 , multimodal form will produce if the frequency is above the first order modal cutoff frequency, and affect the detection accuracy of lamb wave. So according to the dispersion property, we need select the reasonable incentive parameters and sensing element to reduce the modes, and improve detection accuracy of the lamb wave. The dispersion curves associated with the propagation of Lamb waves in the organic glass having the same mechanical and geometric properties of the test samples used in this study are presented in Fig. 1, in which the modes S0 and A0 at $0.5 \mathrm{MHzmm}$ are highlighted by blue curve and red curve respectively.

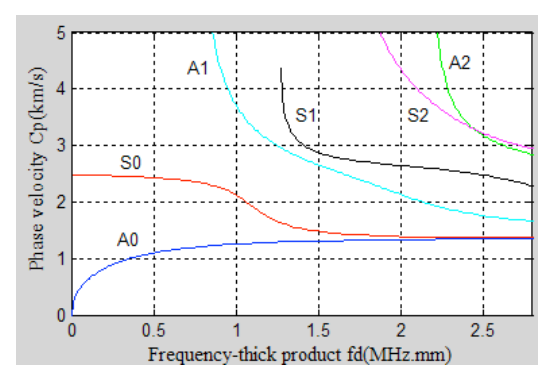

a

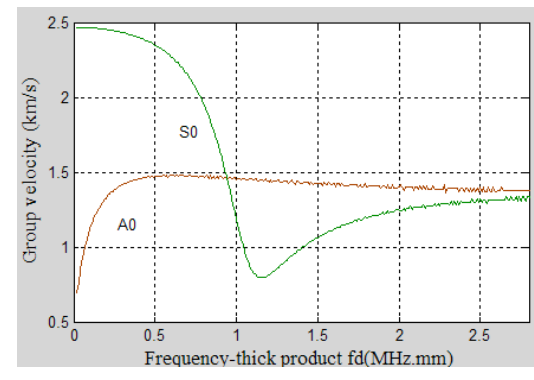

$\mathrm{b}$

Fig. 1. Dispersion curves of the organic glass plate a-phase velocity; b-group velocity

Elastic waves that propagate between two parallel surfaces of thin solid plates with free boundary are known as Lamb waves or guided waves. Lamb waves propagate both as symmetric and anti-symmetric modes, and the number of these modes depends on the product of the excitation frequency and the plate thickness. In order to further study the interaction mechanism of various defects in Lamb wave and structure, we can achieve a single mode of double excitation. The following is a dual excitation method for the realization of the piezoelectric chip, the two piezoelectric chips with the same performance are arranged on the upper and lower surfaces of the plate. When the voltage signals with the same amplitude and phase are applied, the single S0 mode can be excited. While the voltage signal with the opposite phase of the same amplitude is applied, a single A0 mode can be obtained. Figure 2 shows the piezoelectric wafer - plate system, whose middle surface is the middle surface of the plate. That is to say, the vibration of the particle in the plate is caused by double piezoelectric wafers after electric energy and mechanical energy conversion on the full symmetry. The stress and strain of the particles are also about the symmetry of the 
surface. The stress of each particle such as is shown in the formula (1) and the formula (2)[8] .

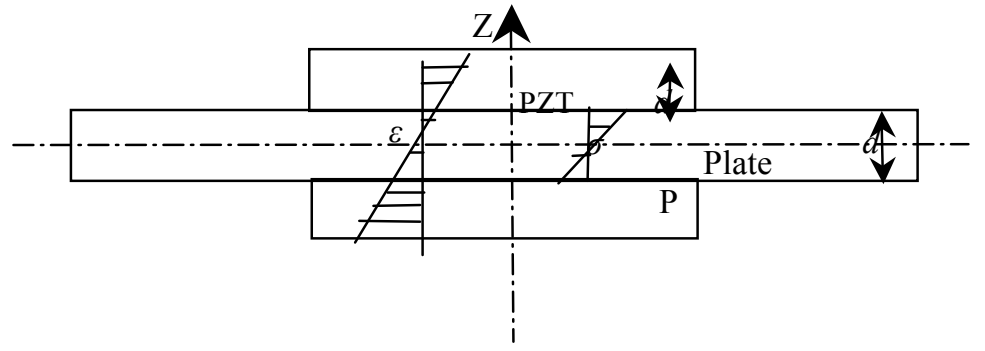

Fig. 2. Excitation principle diagram of double piezoelectric wafers

$$
\begin{gathered}
\sigma_{x 1}=\frac{2 \sigma_{1}^{i}}{d_{1}} z, 1 \leq z \leq \frac{d_{1}}{2} \\
\sigma_{x 2}=A \frac{E_{2}}{E_{1}} \frac{2 \sigma_{1}^{i}}{d_{1}} z-\frac{E_{2}}{1-v_{2}} \prod, 1 \leq z \leq d_{2}
\end{gathered}
$$

Where $\sigma_{x 1}, \sigma_{x 2}$ are the stress of the plate and PZT; $E_{1}, E_{2}$ are the elastic modulus of the plate and PZT respectively $v_{2}$ is Poisson's ratio. And the strain between the plate and the PZT produced by excitation signal is obtained as followed.

$$
\varepsilon_{x}=\frac{d_{31} V}{d_{2}}
$$

When the voltages are applied on the upper and lower of two piezoelectric chips to make simultaneous radial vibration, which can produce symmetric fluctuations by driving the particles on the plate. In this way, the Lamb mode S0 wave is generated. On the contrary, when the voltage is applied to make simultaneous reverse vibration, which can produce symmetric fluctuations in the opposition, and the Lamb mode A0 wave is obtained.

\subsection{Demodulation of the reception principle}

Sensing principle of FBG is based on the Bragg wavelength reflection or transmission spectrum detection. In general, the speed range of fiber Bragg grating demodulation devices is $1 \mathrm{kHz}-4 \mathrm{kHz}$, which do not enough to satisfy the high demodulation speed for ultrasonic signals. So the fiber grating demodulation system need adopt new demodulation methods. The following demodulation system is one of these methods which can satisfy this condition, as shown in figure 2 . The wavelength demodulation system for the FBG sensor is composed of a wavelength tunable laser, an optical coupler and a photodetector. The tunable laser as a light source inspires a beam of light to the FBG through an optical coupler. Then the reflected light from the FBG 
propagates to the photodetector through the coupler. If the central wavelength of the laser beam is tuned to be a little shorter than that of the FBG at free strain, the power relationship between laser and FBG is shown in figure 3[8][9].

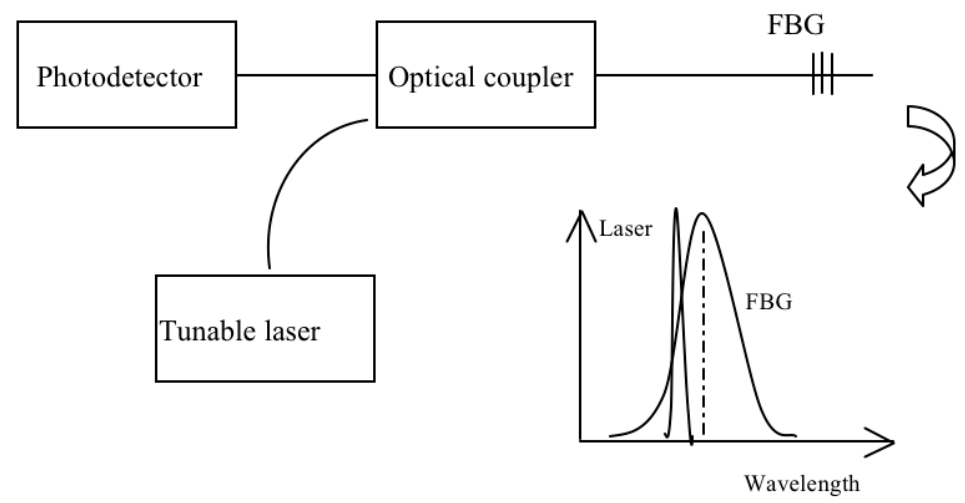

Fig. 3. The demodulation principle of FBG sensor

The optical intensity received by the photodetector is given as following:

$$
P=M_{1} \int_{-\infty}^{\infty} R_{S}(\lambda) S(\lambda) d \lambda
$$

where $R_{s}(\lambda)$ is the reflectivity function of the FBG, $S(\lambda)$ is the spectrum function of the laser source, $M_{1}$ is the efficiency for light energy utilization, which can be regarded as constant if no energy loss. The reflection spectrum of the FBG sensor can be modeled as a Gaussian type to simplify the analysis according to the related model .And the laser beam can also be modeled as Gaussian Beam. So the optical power of photodetector can be derived as following:

$$
P=\frac{M_{1}}{2} R_{s 0} I_{0} \sqrt{\frac{\pi}{\ln 2}}\left\{\frac{\Delta \lambda_{0} \Delta \lambda_{S}}{\left(\Delta \lambda_{0}{ }^{2}+\Delta \lambda_{S}{ }^{2}\right)^{\frac{1}{2}}} \times \exp \left[-4 \ln 2 \frac{\left(\lambda-\lambda_{S}\right)^{2}}{\Delta \lambda_{S}{ }^{2}+\Delta \lambda_{0}{ }^{2}}\right]\right\}
$$

where $R_{s 0}$ is the maximum reflectivity, $\lambda_{s}$ is the center wavelength of the FBG, $\Delta \lambda_{s}, \Delta \lambda_{0}$ is its spectral full width at half maximum. $I_{0}$ is the peak power, $\lambda_{0}$ is the center wavelength of the laser beam. Therefore, the optical power of photodetector can be obtained according to Eq. 5. The optical power has the maximum if the center wavelength of the FBG equals to that of the laser. When FBG is compressed, there will be little change in wavelength of the FBG in this case. And the overlapped region between the optical power of the laser beam and that of FBG will change along with the intensity of output light accordingly. Thus the wavelength change of the FBG can be measured by the intensity of the photodetector. Therefore, it can analyze the axial strain change of fiber Bragg grating by detecting its wavelength drift, avoiding the influence of electromagnetic field. 


\section{Simulation analysis}

Sugarcane sowing According to the above analysis in part II, in order simplifies the calculation, we assume that one excitation is placed directly on the plate without the adhesive layers. First, a geometric model is built in COMSOL software, model size of the plate is $1000 * 600 * 5 \mathrm{~mm}^{3}$ (as shown in figure 4 and figure 5). The material parameters of the plate are specified: elastic modulus is $6.4 \mathrm{GPa}$, poisson coefficient is 0.3 , density is $1190 \mathrm{~g} / \mathrm{cm}^{3}$.In figure 4 , one excitation signal is applied on ' $\mathrm{P}$ '. Here we use a modulation force directly instead of the piezoelectric excitation effect, whose center frequency is $100 \mathrm{kHz}$, and the amplitudes is $100 \mathrm{~N}$. In this way, the calculation results can be obtained after the mesh processing, such as the acoustic field distribution, from which we can observe the variation of the acoustic field at different time, such as $8 \mathrm{e}^{-5} \mathrm{~s}, 1.3 \mathrm{e}^{-5} \mathrm{~s}$. In these moments wave packets have spread to damage hole, where the interaction produces between them. From figure $4 \mathrm{~b}$, we can see that the hole is as the second source to launch.

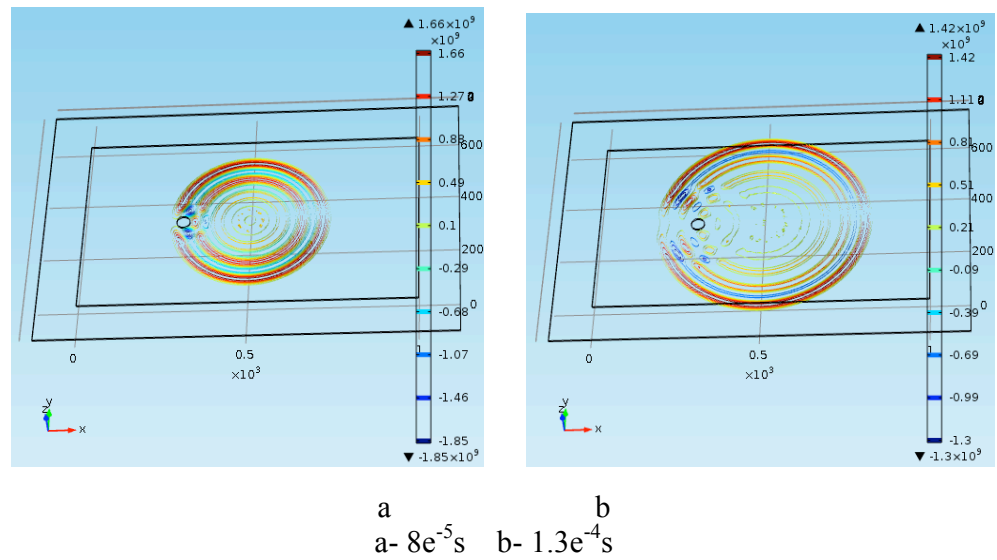

Fig. 4. The acoustic pressure field on at different time

In figure 5a, the points 'A', 'B' ,'C' represent the three different detection positions respectively, and the excitation point ' $\mathrm{P}$ ' is lie on the centre of the plate. ' $\mathrm{X}$ ' represents a circular defect, whose diameters are $10 \mathrm{~mm}$ and thickness is $5 \mathrm{~mm}$ on the vertical lines away from the center of the plate. In figure $5 b, 5 c, 5 d$, the blue curve represents the response of point $\mathrm{A}$, the green curve represents the response of point $\mathrm{B}$, and the red curve represents the response of point $\mathrm{C}$. By comparing the responses of three points, we can see that the amplitudes of the detection signals will have a change owing to the distance between the detection point and excitation point. The distances $\mathrm{CP}$ is the farthest distance, so the detection signal amplitude(as shown in red curve) is smallest due to the attenuation. In addition, from the figure $5 \mathrm{~b}, 5 \mathrm{c}, 5 \mathrm{~d}$ we can see that the distances AP and BP are the same, so the signal arrival time of these two points is the same, whose initial waveforms are coincident and the back waveforms are different due to the defect reflection and boundary reflection. Assuming that the transmis- 
sion speed is $2500 \mathrm{~m} / \mathrm{s}$, AP is $180 \mathrm{~mm}$, so it spends the longest time $0.18 / 2500=72 \mathrm{us}$. This result is consistent with the simulation result, as shown in figure $5 \mathrm{~b}$, in which the arrival time of the first wave packet is $0.8 * 10^{-4} \mathrm{~s}$. Meanwhile, CP is $240 \mathrm{~mm}$, so it spends the longest time $0.24 / 2500=96 \mathrm{us}$, which is approximately consistent with the simulation result $1 * 10^{-4} \mathrm{~s}$. The distance $\mathrm{XB}$ is closer to the injury, which is far away from point $\mathrm{A}$. So the wave packet of point $\mathrm{B}$ reflected by the injury may be submerged in the direct wave packet. Overall, the simulation results(as shown in figure $5 \mathrm{c}, 5 \mathrm{~d})$ show that the wave number of the modulation wave is more, the energy is stronger. Thus it is easy to cause the signal aliasing and is more difficult to further identify the damage signal. So we need to select the modulation wave number.

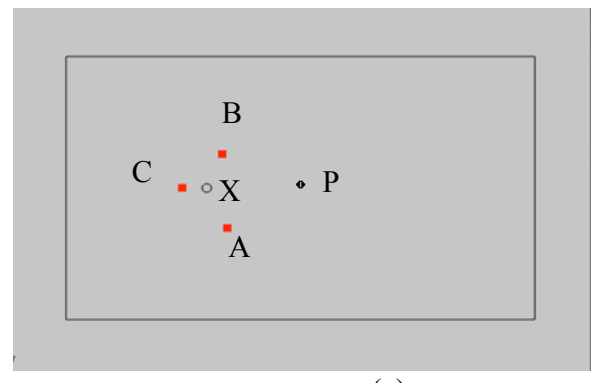

(a)

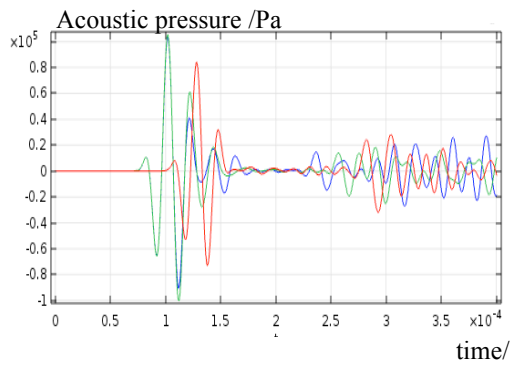

(c)

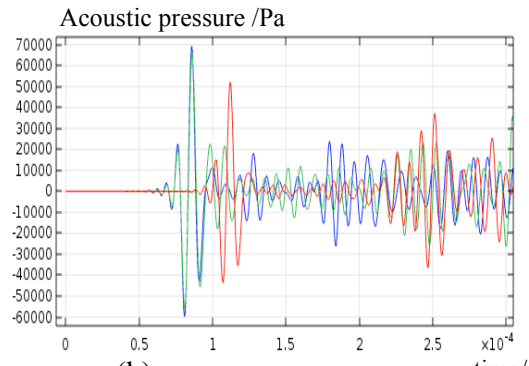

(b)

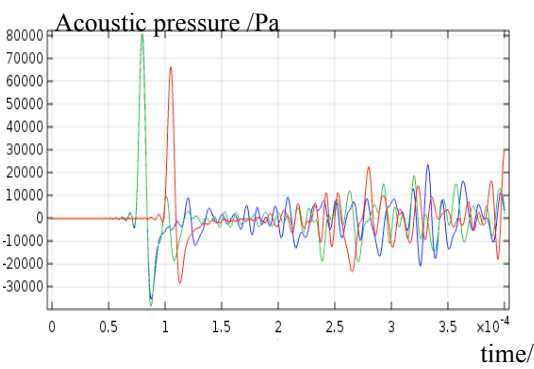

(d)

a- Monitoring point arrangement ; b-Three peak modulation wave with $100 \mathrm{kHz}$; c-Three peak modulation wave with $50 \mathrm{kHz}$; d-One peak modulation wave with $50 \mathrm{kHz}$

Fig. 5. The acoustic pressure field under different incentives

\section{Experiment based on FBG sensor}

The Ultrasound strain on the metal plate is usually only a few microstrains, so the fiber grating demodulation system should satisfy the high demodulation speed to measure ultrasonic signals ensuring accuracy at the same time. In order to demonstrate high sensitivity of the cross modulation effect to the defects in a plate sample, we use adjustable laser edge filter demodulation as new demodulation system. Principle diagram is shown in figure 6 (a), where piezoelectric wafers are used for the excitation, while FBG sensors are used for detection response. The tuning range of tuna- 
ble laser light source with typical line width of $200 \mathrm{kHz}$ is $1260-1630 \mathrm{~nm}$. And the object of study is organic glass plate whose dimension is $1000 \mathrm{~mm} * 600 \mathrm{~mm} * 5 \mathrm{~mm}$, and incentive layout is shown in figure $6(\mathrm{~b})$.

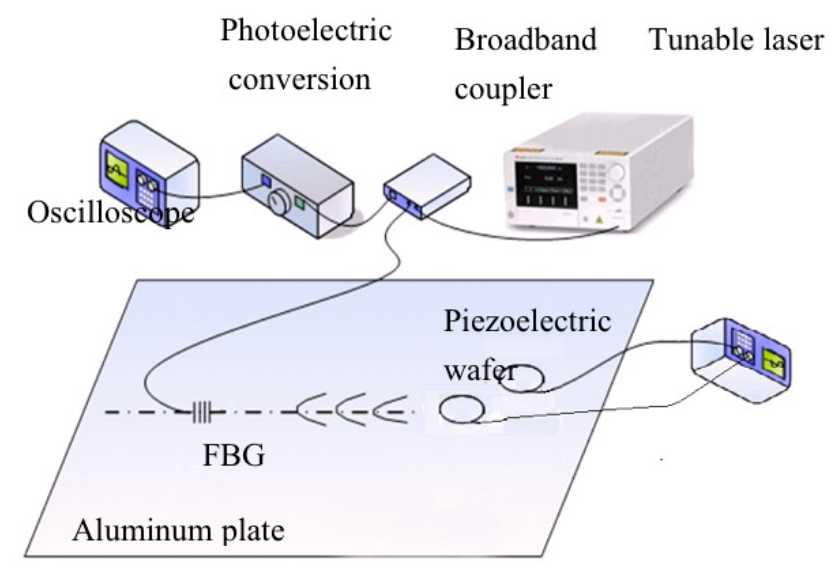

(a) Principle diagram

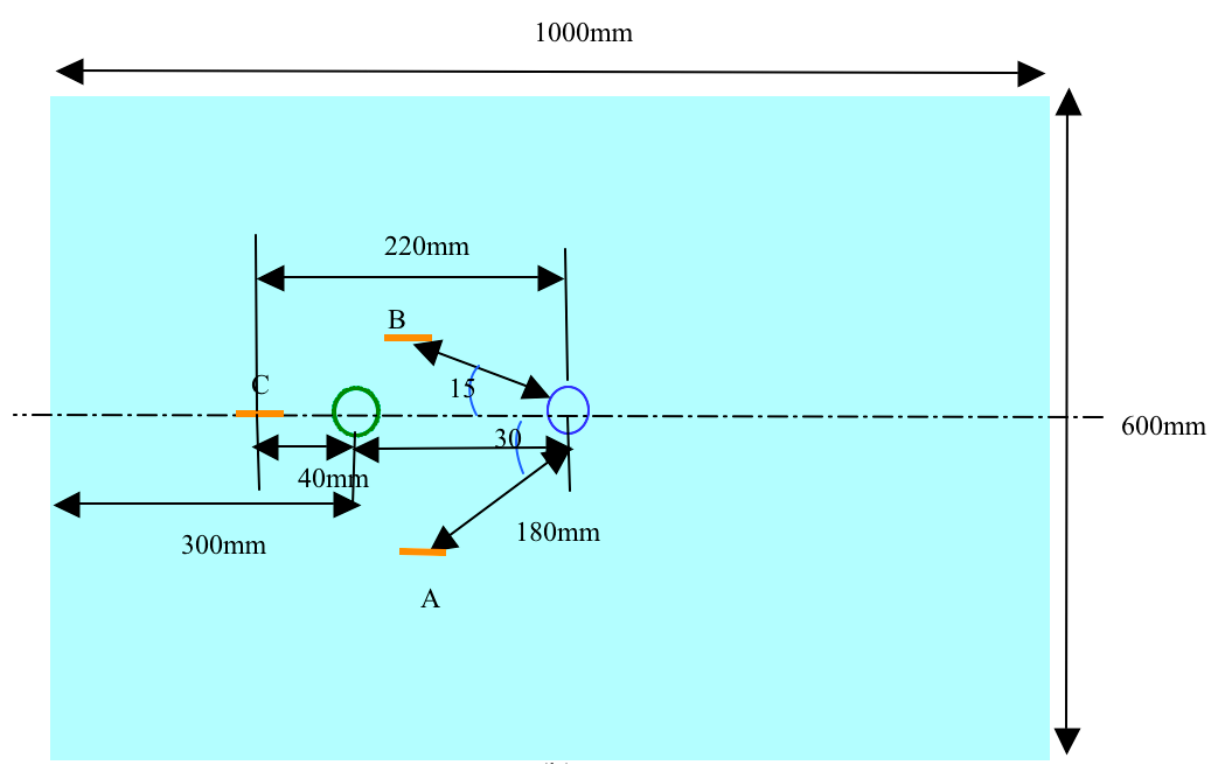

(b) Incentive layout

Fig. 6. xperimental equipment

In figure 6 (b), blue circle represents the piezoelectric wafers with diameter $10 \mathrm{~mm}$ while green circle represents the damage hole with diameter $10 \mathrm{~mm}$. Orange horizontal lines 'A', 'B', 'C' represent FBG sensors with the length of $5 \mathrm{~mm}$, whose wavelengths are $1315,1300,1293 \mathrm{~nm}$ respectively. Here the ultrasonic frequency is not 
more than $100 \mathrm{kHz}$, so the length of FBG with $5 \mathrm{~mm}$ can satisfy with this condition. Due to the temperature influence, we keep temperature 20 degrees based on the temperature measurement during the experiment. In this way, it can eliminate the influence of temperature factor. Due to the strain sensitivity of the optical fiber grating and the acoustic axis of the ultrasonic energy, we firstly study the basic characteristics of FBG sensors in ultrasonic excitation with different incentive parameters. On the basis of this, we can further study the response analysis of FBG under multi-excitation and subsequent damage assessment. The relevant parameters such as wavelength and position parameters of FBG sensors are as shown in table 1.

Table 1. Wavelength and position parameters of FBG sensors

\begin{tabular}{|l|c|c|c|}
\hline \multicolumn{1}{|c|}{ Comparison } & A & B & C \\
\hline FBG wavelength $(\mathrm{nm})$ & 1293 & 1315 & 1300 \\
\hline Distance from source $(\mathrm{mm})$ & 180 & 180 & 240 \\
\hline Distance from damage $(\mathrm{mm})$ & 90 & 40 & 40 \\
\hline
\end{tabular}

\subsection{Response analysis of FBG on different positions}

We know that under single excitation it will appear at least two modes of the wave packet, S0 and A0. Here, in order to facilitate the detection, the wave packet can be suppressed by inhibiting one of the modes allowing a single mode wave packet, as shown in figure 7, in which response waveforms of different points A, B, C in different excitation modes are compared. According to the simulation results, excitation source is used by half-peak modulated wave, whose frequency is $100 \mathrm{kHz}$ and the voltage amplitude is $150 \mathrm{~V}$ (as shown in figure 8). Figure 7(a) is one-side incentive and figure $6 \mathrm{~b}$ is asymmetric incentive. It can be seen from figure 7 that the peak times of the detection point A are basically same as that of the detection point B and the magnitudes are roughly equivalent. According to figure 7(b) and table 2, we can draw the conclusion that the waveform pattern of the symmetrical excitation is relatively simple, and the amplitude of the S0 model is significantly higher than that of single side excitation, which is nearly 2 times.

According to the frequency dispersion characteristic curve, we can get the speed parameters. When the excitation frequency is $100 \mathrm{k}$ and the thickness of the glass plate is $5 \mathrm{~mm}$, the frequency thick product parameter is $0.5 \mathrm{MHz} \cdot \mathrm{mm}$. So the corresponding speed is respectively as follows: $V_{S 0}=2349 \mathrm{~m} / \mathrm{s}, V_{A 0}=1474 \mathrm{~m} / \mathrm{s}$. According to the detection distance in table 1 , the time can be calculated respectively as followed in table 3 . 


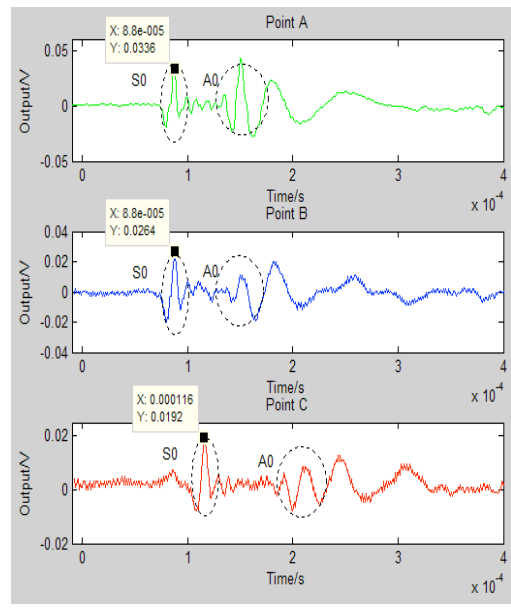

(a) Single-side incentive

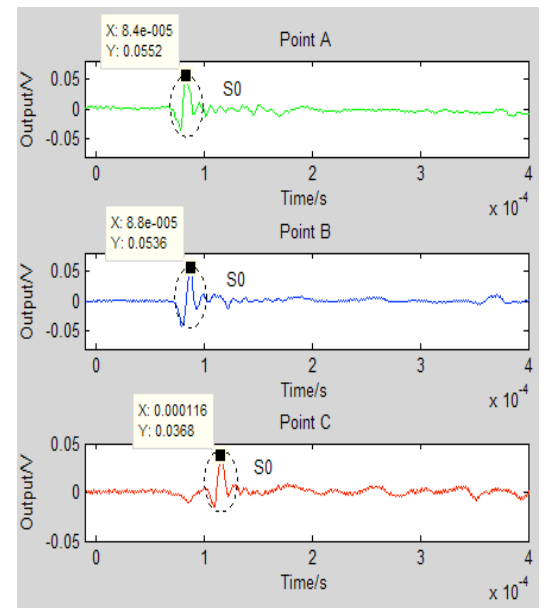

(b) asymmetric incentive

Fig. 7. Response on different positions under different incentives

Table 2. Test amplitude of FBG sensors on different positions

\begin{tabular}{|l|c|c|c|}
\hline \multicolumn{1}{|c|}{ Detection peak(mV) } & A & B & C \\
\hline \multirow{2}{*}{ Single-side incentive } & 33.6 & 26.4 & 19.2 \\
\cline { 2 - 4 } & 40.8 & 11.2 & 6.4 \\
\hline asymmetric incentive & 55.2 & 53.6 & 36.8 \\
\hline Antisymmetry incentive & 40.8 & 34.4 & 14.4 \\
\hline
\end{tabular}

Table 3. Test time of FBG sensors on different positions

\begin{tabular}{|l|c|c|c|c|c|c|c|c|c|}
\hline \multirow{2}{*}{$\begin{array}{c}\text { Detection } \\
\text { times }\end{array}$} & \multicolumn{4}{|c|}{ A } & \multicolumn{4}{c|}{ B } & \multicolumn{3}{c|}{ C } \\
\cline { 2 - 10 } & Test & $\begin{array}{c}\text { Calcula- } \\
\text { tion }\end{array}$ & Error & Test & $\begin{array}{c}\text { Calcula- } \\
\text { tion }\end{array}$ & Error & Test & $\begin{array}{c}\text { Calcula- } \\
\text { tion }\end{array}$ & Error \\
\hline $\begin{array}{l}\text { asymmetric } \\
\text { incentive }\end{array}$ & 78 & 77 & $1 \%$ & 82 & 77 & $6 \%$ & 110 & 93 & $18 \%$ \\
\hline $\begin{array}{l}\text { Antisymmetry } \\
\text { incentive }\end{array}$ & 128 & 122 & $5 \%$ & 127 & 122 & $4 \%$ & 170 & 150 & $13 \%$ \\
\hline
\end{tabular}

In the process of the experiment, the initial excitation signal has a delay time. So the experimental detection time is needed to subtract the delay time of the excitation source $6 \mathrm{e}^{-6} \mathrm{~s}$. After subtracting the delay time it can be used as the test time. By comparing the test time and Calculated time in table 3 , we can see that time errors of detection points A, B are small. The errors may be caused by the deviation of the detection range or the estimated speed, which are within the normal range. But time errors of detection point $\mathrm{C}$ are too big. Maybe the defect hole affects the normal transmission of the wave, resulting in the different estimation of the velocity. Overall, Point $\mathrm{C}$ is relatively farthest and damage reflection signal is weak through the defect hole. But it still follows the law: the amplitude of the symmetric mode under symmetric excita- 
tion is higher than that of the single side excitation. So it is not suitable for testing point. In addition, point $\mathrm{B}$ is closer to the damage, so damage signals are likely to be submerged in the direct wave packet easily. This conclusion is in agreement with the simulation results. So next we mainly focus on point A to extract damage information.

\subsection{Response analysis of FBG under different frequencies}

Figure 8 is the responses of the point $A$ under different frequencies, such as $60 \mathrm{kHz}$, $80 \mathrm{kHz}$. Black curve indicates incentive, green curve represents the response under single incentive, blue curve represents the response under symmetric incentive, and red curve is for the antisymmetric excitation response.

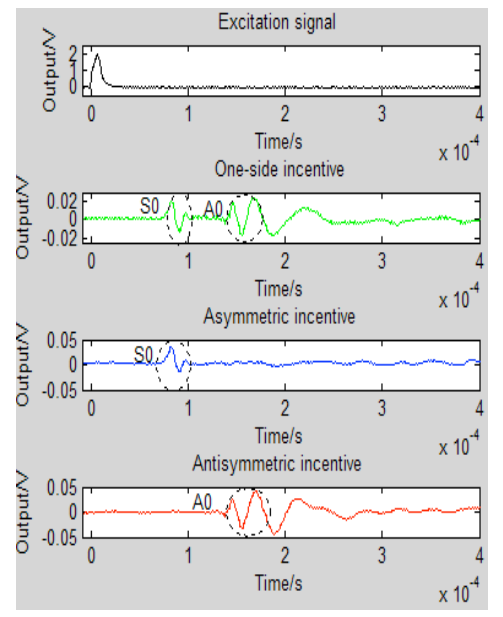

(a) with the frequency $60 \mathrm{kHz}$

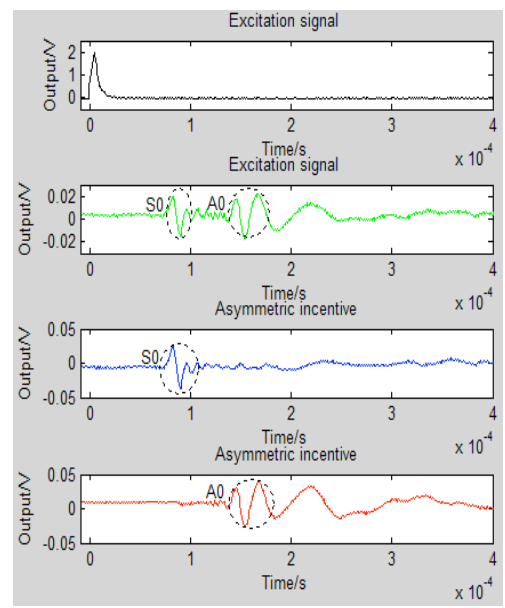

(b) with the frequency $80 \mathrm{kHz}$

Fig. 8. Response of detection point $A$ at different frequencies

Figure 9 is responses of detection point $\mathrm{A}$ at different frequencies after filtering. According to the waveforms as shown in figure 8 and 9, under the antisymmetry incentive with the frequency $60 \mathrm{kHz}$ or $80 \mathrm{kHz}$, A0 mode and $\mathrm{S} 0$ mode have reflected wave packet, but these reflective waves reflected by the damage are very small. So it is appropriate to compare the relative changes. In this case, here S0 mode has better detection effect than A0 mode.

By removing noise, we can clearly see the wave packet reflected by the damage. From figure 9a, the reflected wave packet represented by red curve in antisymmetric mode is basically invisible, which is not suitable for damage detection. While the corresponding reflected wave packet with blue curve in symmetry mode is relatively large, this is more suitable for damage detection. Similarly, the same conclusion applies to figure $9 \mathrm{~b}$. From figure 9 , we can see that the reflection wave packet with blue curve is relatively larger in the symmetry mode, which is suitable for damage detection. 


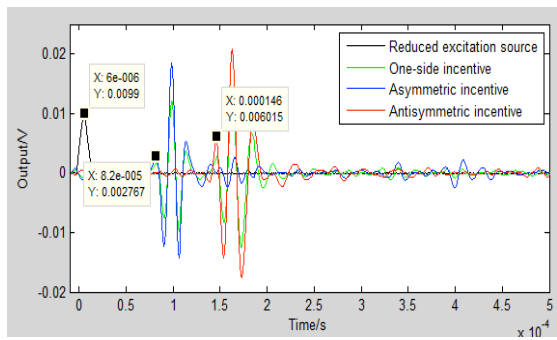

(a) with the frequency $60 \mathrm{kHz}$

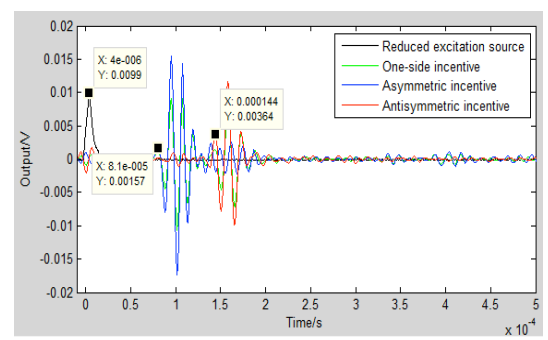

(b) with the frequency $80 \mathrm{kHz}$

Fig. 9. Response at different frequencies after filtering

\section{Conclusion}

This work focuses on the single-mode ultrasonic Lamb wave technique for crack detection by FBG, which is combined the advantage of fiber grating sensing technique. In this paper, taking the plate structure as the object, a new attempt by singlemode ultrasonic Lamb detection with FBG sensors has been investigated. First, single-mode ultrasonic Lamb technique and the strain characteristics of FBG sensors are introduced. Second, the simulation analysis of the ultrasonic Lamb wave signals are carried out and time domain simulation of the plate structure are performed, which reveal various ultrasonic Lamb wave signals on different detection positions. Third, the experimental system based on ultrasonic Lamb wave technique-fiber Bragg grating detection is built and response analysis of FBG sensors under the different excitations is given. The results reveal an extremely efficient single-mode ultrasonic Lamb wave technique. And it shows that this single-mode ultrasonic Lamb wave techniqueFBG sensing system is feasible and it is sensitive to crack damage by comparison of the signals in healthy area and damaged area.

\section{Acknowledgment}

This work is supported the following fund:

2017 The project of Hubei Province Natural Science Foundation for Youths (No.2017CFC890).

2017 the Science Research Project of Hubei Province Education Department (No.B2017298).

\section{$7 \quad$ References}

[1] Wilcox P D, Lowe M J S, Cawley P: The effect of dispersion on long-range inspection using ultrasonic guided waves[J]. NDT\&E International, 34 (1),1 9, (2001). https://doi.org/10.1016/S0963-8695(00)00024-4 
Paper-Damage Detection in a Plate Structure based on FBG sensing Technique under a Single-Mode...

[2] Wieslaw Ostachowicz, PawelKudela, PawelMalinowski, TomaszWandowski: Damage localisation in plate-like structures based on PZT sensors. Mechanical Systems and Signal Processing ,vol.23, 1805-1829, (2009). https://doi.org/10.1016/j.ymssp.2008.10.011

[3] Kim S B, Sohn H: Instantaneous reference-free crack detection based on polarization characteristics of piezoelectric materials[J]. Smart Materials and Structures, 16(6): 2375 2387,(2007). https://doi.org/10.1088/0964-1726/16/6/042

[4] Su Z, Ye L: Selective generation of Lamb wave modes and their propagation characteristics in defective composite laminates[J]. Journal of Materials: Design and Applications, 218(2), 95 110, (2004). https://doi.org/10.1109/TUFFC.2009.1201

[5] Clarke T, Simonetti F, Rohklin S et al. Development of a low-frequency high purity A0 mode transducer for SHM applications[J]. IEEE Transactions on Ultrasonics, Ferroelectrics, and Frequency Control,56(7), 1457 1468, (2009).

[6] Wang D, Ye L, Lu Y, et al: A damage diagnostic imaging algorithm based on the quantitative comparison of Lamb wave signals[J]. Smart Materials and structures, 19(6), 065008$1 \sim 12,(2010)$.

[7] Michaels J E: Detection, location and characterization of damage in plates with an in situ array of spatially distributed ultrasonic sensors[J]. Smart Materials and Structures, 17(3), 035035-1 15, (2008).

[8] Xiaole Liu, Houguang Liu, Jianhua Yang, Grzegorz Litak,Gang Cheng, Shuai Han: Improving the bearing fault diagnosis efficiency by the adaptive stochastic resonance in a new nonlinear system. Mechanical Systems and Signal Processing. 58-76,(2017). https://doi.org/10.1016/j.ymssp.2017.04.006

[9] Aldo Baccigalupi, Annalisa Liccardo: The Huang Hilbert Transform for evaluating the instantaneous frequency evolution of transient signals in non-linear systems. Optik International Journal for Light and Electron Optics. 3417-3422, (2016)..

[10] Irene Buj-Corral, Jesús Álvarez-Flórez, Alejandro Domínguez-Fernández: Acoustic emission analysis for the detection of appropriate cutting operations in honing processes. Mechanical Systems and Signal Processing. 873-885, (2018).

\section{Authors}

Cai Li (1980-), come from Wuhan, Hubei province. Ph.D.degree, the main research direction for equipment detection and diagnosis.(e-mail: caii_whut@163.com, affiliation: School of Mechanical and Electronic Engineering, Wuhan Donghu University, Wuhan,430070, China).

Huang Xiao (1982-), come from Wuhan, Hubei province. Ph.D.degree, the main research direction for fiber Bragg grating detection technology.(e-mail: 51277619@qq.com, affiliation: School of Mechanical and Electronic Engineering, Wuhan University of Technology, Wuhan, 430070, China).

Zuo Xiaoqiong (1978-), come from Wuhan, Hubei province. master degree, the main research direction for electrical engineering management.(e-mail: 32257299@qq.com, affiliation: School of Mechanical and Electronic Engineering, Wuhan Donghu University, Wuhan, 430070, China).

Article submitted 12 January 2018. Resubmitted 20 February 2018. Final acceptance 23 April 2018. Final version published as submitted by the authors. 